\title{
Anisotropic Coulomb Explosion of Acetylene and
}

\section{Diacetylene Derivatives}

Naoya Mitsubayashi ${ }^{\mathrm{a}}$, Tomoyuki Yatsuhashi ${ }^{\mathrm{a}, \mathrm{b},{ }^{*}}$, Hiroki Tanaka ${ }^{\mathrm{a}}$, Satoshi Furukawa ${ }^{\mathrm{a}}$, Masatoshi Kozaki ${ }^{\mathrm{a}}$, Keiji Okada $^{\mathrm{a}}$, Nobuaki Nakashima ${ }^{\mathrm{a}}$

${ }^{a}$ Department of Chemistry, Graduate School of Science, Osaka City University, 3-3-138 Sugimoto, Sumiyoshi, Osaka 558-8585 Japan

${ }^{\mathrm{b}}$ PRESTO, Japan Science and Technology Agency (JST), 4-1-8 Honcho Kawaguchi, Saitama, 3320012 Japan

Keywords: Femtosecond laser; High intensity laser; Multiply charged ions

* Corresponding author at: Department of Chemistry, Graduate School of Science, Osaka City University, 3-3-138 Sugimoto, Sumiyoshi, Osaka 558-8585, Japan. Tel.:+81 666052554

E-mail address: tomo@sci.osaka-cu.ac.jp (T. Yatsuhashi) 

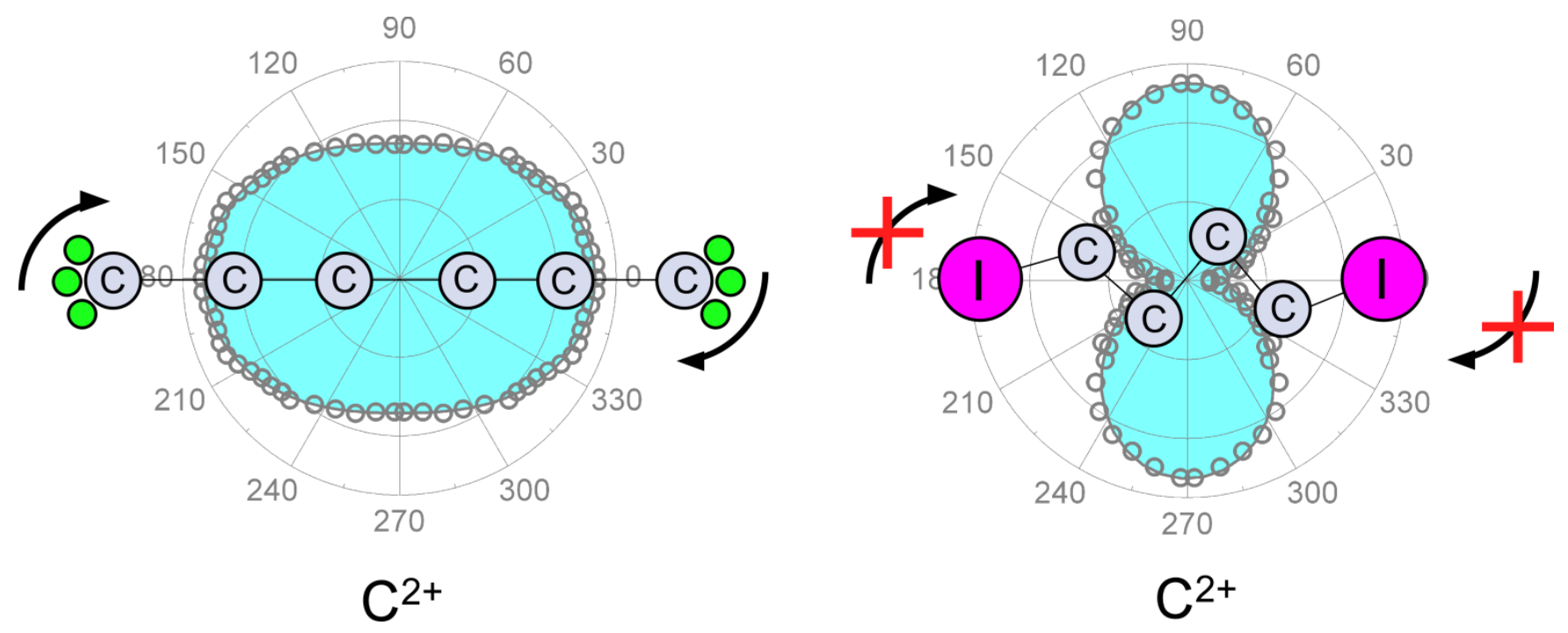

Graphical Abstract 


\begin{abstract}
We investigated the significant differences in the angular distribution of carbon ions ejected from dimethylacetylenes $\left(\mathrm{CH}_{3}-\mathrm{C} \equiv \mathrm{C}-\mathrm{CH}_{3}\right.$ and $\left.\mathrm{CH}_{3}-\mathrm{C} \equiv \mathrm{C}-\mathrm{C} \equiv \mathrm{C}-\mathrm{CH}_{3}\right)$ and diiodoacetylenes (I-C $\equiv \mathrm{C}$-I and I$\mathrm{C} \equiv \mathrm{C}-\mathrm{C} \equiv \mathrm{C}-\mathrm{I})$ induced by a Coulomb explosion. The longest linear chain molecule ever reported was exposed to intense femtosecond laser fields $\left(40 \mathrm{fs},<5 \times 10^{14} \mathrm{Wcm}^{-2}\right)$ in this study. In the cases of the diiodoacetylenes, the angular distributions of the carbon ions were orthogonal with respect to the laser polarization direction and became narrower as the length of the molecules became longer. This is in sharp contrast to the dimethylacetylenes, in which the angular distributions of the carbon ions were parallel with respect to the laser polarization direction and became broader as the length of the molecules became longer. The specific angular distributions of carbon ions ejected from the diiodoacetylenes are explained in terms of the frozen molecular rotation, the blocking of carbon ion emission, and the bending of carbon chains at highly charged states due to the presence of iodines.
\end{abstract}




\section{Introduction}

During the past several decades, the interactions between molecules and high-intensity laser fields have been extensively studied [1]. Alignment, structural deformation, and multiple ionizations occur due to strong electric fields [2]. Chemical reactions of multiply charged ions [3] that are efficiently formed in such fields are quite interesting because of their electron-deficient nature, spin multiplicity variations, charge-dependent channels, and Coulomb explosions. The reactions of dications, which were first produced by a conventional electron impact ionization method, have been investigated by mass spectrometry [4]. Dications sometimes have a relatively long lifetime that allows structural deformation and rearrangement before a Coulomb explosion (a charge separation process).

For example, phenyl acetylene dication dissociates into $\mathrm{CH}_{3}{ }^{+}$and $\mathrm{C}_{7} \mathrm{H}_{3}{ }^{+}$after the structural rearrangement [5]. From the kinetic energy releases of the reactions, the distance between the two positive charges before dissociation has been estimated to be $7.3 \AA$ [5]. Thus the linear chain allene-type structure, in which the two positive charges are separated from each other, has been considered to be an intermediate before the Coulomb explosion [5]. In contrast, the multiply charged molecular ions formed in femtosecond laser fields show unique behavior. The angular distribution of $\mathrm{CD}^{+}$ejected from the acetylene moiety of phenyl acetylene- $d$ shows strong anisotropy along the laser polarization direction, thus $\mathrm{CD}^{+}$is ejected before molecular rotation occurs [6]. Recent ultrafast pump and probe mass spectroscopy studies revealed the ultrafast migration (90 fs) and recurrence (300 fs) of hydrogen within the framework of acetylene dication under the influence of intense laser fields [7]. Ultrafast hydrogen migration in intense laser fields has been investigated in a variety of molecular ions, such as acetonitrile [8], methanol [9], butadiene [10], allene, [11] and methylacetylene [12].

It is known that there is a strong relationship between the ion emission direction and the laser polarization direction (linear polarization) when a Coulomb explosion is induced in the intense laser fields [13]. The ion dynamics have been successfully investigated by covariance mapping [14], momentum imaging techniques [15], and the simple consideration of kinetic energy releases. Much interesting information about the deformation of molecules in intense laser fields has been demonstrated 
[16]. The degree of I-I bond elongation in a stretching coordinate before a Coulomb explosion at $10^{15}$ $\mathrm{Wcm}^{-2}$ depended on the charge of iodine [17]. A deformation along the bending coordinates before a Coulomb explosion has been found for carbon dioxide, which has a linear structure in the neutral state [15]. However, most of the molecules examined in this context have been two- or three-atom molecules such as $\mathrm{CO}_{2}$ [15], $\mathrm{SO}_{2}$ [18], and $\mathrm{SCO}[19]$.

We have investigated the anisotropic Coulomb explosion of benzene as a typical planer-shape molecule [20, 21], and fullerene as a typical sphere-shape molecule [22] at $10^{16} \mathrm{Wcm}^{-2}$, and the influence of laser electric fields on the carbon ion emission has been demonstrated by molecular dynamics (MD) simulation. Neutral benzene has a planar structure with $\mathrm{D}_{6 \mathrm{~h}}$ symmetry; however, our MD simulation showed that the precursor ion has a ladder-like shape along the laser polarization direction before the Coulomb explosion [20, 21]. The angular distribution of carbon ions exploded from fullerene was like a rugby-ball-shape with the long axis parallel to the laser polarization direction, although neutral fullerene has a spherical structure [22]. In contrast, acetylene, the most representative four-atom linear molecule, maintained the same symmetry as that of the neutral ground state before a Coulomb explosion [23], and carbon ions were sharply ejected along the laser polarization direction.

We have demonstrated the mass effects on the angular distribution of carbon ions ejected from acetylene and diiodoacetylene, which have similar geometry but different terminal atom masses [24]. Carbon ions originated from diiodoacetylene are emitted in an orthogonal direction, whereas those from acetylene are emitted in a parallel direction with respect to the laser polarization direction. We have explained the distinct carbon ion emissions from diiodoacetylene in terms of the persistence of a molecular frame at a high charge state, and the deformation within the Coulomb field formed by the two adjacent iodine ions [24]. This finding was confirmed by the coincidence study for the Coulomb explosion of diiodoacetylene induced by the collision with high-energy projectiles $\left(\mathrm{Ar}^{8+}\right)$ [25]. However, it is not yet certain whether the mass effect is general and applicable to other substituents and also longer linear chain molecules. 
In the present study, we prepared four acetylene and two diacetylene derivatives with different terminal substituents $\left(\mathrm{H}, \mathrm{CH}_{3}, \mathrm{I}\right)$ and different numbers of carbon atoms in linear carbon chain (from two to six). We investigated the angular distributions of atomic ions and $\mathrm{CH}_{3}{ }^{+}$. Our results revealed that the direction of the carbon ion emission changes from parallel to orthogonal with respect to the laser polarization direction when the terminal hydrogen or $\mathrm{CH}_{3}$ is replaced by iodine. In addition, the angular distributions of carbon ions became broader as the length of molecule became longer in the cases of acetylene, dimethylacetylene, and dimethyldiacetylene, whereas the angular distributions of carbon ions of diiodoacetylene and diiododiacetylene became narrower. We discuss the opposite trends in the angular distribution of carbon ions in terms of the molecular structure deformation, original location of the carbons, and the degree of molecular rotation.

\section{Materials and methods}

The molecules examined in this study are shown in Fig. 1. Acetylene (Takachiho, 99.9\%) was used without further purification. 2-Butyne (dimethylacetylene, DMA, Sigma-Aldrich, 99\%) and 2, 4hexadiyne (dimethyldiacetylene, DMDA, Tokyo Kasei, 98\%) was purified by vacuum distillation. Diiodoacetylene (DIA) [26], diiododiacetylene (DIDA) [27], and 2-iodo-1-phenylacetylene (IPA) [28] were prepared as described. The samples other than those of acetylene were degassed before use by repeated freeze-thaw cycles. Xenon was purchased from Japan Air Gases with the stated purity of 99.99 $\%$

Briefly, the multiple ionization of acetylene derivatives and xenon was carried out with a 40 -fs pulse centered at $0.8 \mu \mathrm{m}$, and the ions were detected by a linear time-of-flight mass spectrometer. An all-diode pumped Ti:Sapphire laser (Alpha 100/1000/XS hybrid, <30 fs, 100-Hz, >15 mJ, $0.8 \mu \mathrm{m} ;<30$ fs, $1-\mathrm{kHz},>2 \mathrm{~mJ}, 0.8 \mu \mathrm{m}$; Thales Laser) was used. The schematic of the experimental set-up is shown in Fig. 2a. The pulse width was measured by a second-order single-shot autocorrelator (SSA, TAIGA; Thales Laser). The laser beam passes through several materials such as a beam splitter (BS, quartz, antireflection coated, $t=1 \mathrm{~mm}$ ), a zero-order half-wave plate (HWP), a focusing lens (quartz, anti-reflection 
coated, $t=5 \mathrm{~mm}$ ), and an ionization chamber window (W, quartz, $t=3 \mathrm{~mm}$ ). The same materials were placed in front of the SSA. A quartz plate (SQ) with the same thickness was used instead of a lens. Dielectric mirrors (M) were used to reflect the laser beam. The group velocity dispersions introduced by these materials were compensated with acoustoptic programmable dispersive filters (Dazzler; Fastlite) to obtain the minimum pulse width. We did not achieve gain narrowing compensation by the Dazzler, and the typical pulse width was 40 fs.

The laser beam was focused into the ionization chamber with a plano-convex quartz lens of 200$\mathrm{mm}$ focusing length. The position of the lens along the laser propagation direction was adjusted to have a maximum signal intensity of the highest charge state of xenon observed $\left(\mathrm{Xe}^{5+}\right)$. The laser energy was attenuated by the combination of a half-wave plate and plate polarizers before the pulse compressor. A part of the laser beam was reflected by a BS at a small incident angle, and we measured the laser pulse energy using a spectralon-coated integrating sphere (Labsphere) and a calibrated Si pin-photodiode. We determined the actual laser intensity of the linear polarized pulse at the focus by measuring the saturation intensity, $I_{\text {sat }}$ of xenon $\left(1.1 \times 10^{14} \mathrm{~W} \mathrm{~cm}^{-2}\right.$ for a $45-\mathrm{fs}$ pulse) by the method of Hankin et al. [29], and the error in the determination of absolute laser intensity was approx. $\pm 10 \%$. The ions of the acetylene derivatives were measured successively after the measurement of $I_{\text {sat }}$ of xenon without changing experimental conditions between runs.

The direction of the laser polarization $(0.8 \mu \mathrm{m}$, linear) against the ion flight axis was changed by a HWP, and we confirmed the direction by using a broadband polarizing cube beam splitter. The angle measured with respect to the laser polarization direction from the ion flight axis to the detector was defined as $\theta$ (Fig. 2b). When the laser polarization direction was parallel $\left(\theta=0,180^{\circ}\right)$ or perpendicular $\left(\theta=90,270^{\circ}\right)$ to the ion flight axis, this situation is referred to as "parallel" or "orthogonal," respectively. The data were measured for the four quadrants with $4^{\circ}$ steps experimentally, then averaged to improve the ratio of signal intensity to noise intensity, because all of the quadrants were almost identical. We then used the averaged data to make an angular distribution as a polar plot for clearer presentation. 
A linear mode of reflectron-type time-of-flight mass spectrometer (KNTOF-1800; TOYAMA) was used for the ion analysis. The chamber pressure was monitored $20 \mathrm{~cm}$ away from the laser focus point with a cold cathode pressure gauge. The base pressure of the ionization chamber and the time-of-flight chamber without sample gas was below $5 \times 10^{-7} \mathrm{~Pa}$. Vapor samples were introduced effusively by a precision valve. The sample pressure in the ionization chamber was kept below $5.0 \times 10^{-5} \mathrm{~Pa}$ during the experiments to avoid the space-charge effect. The pressure of the time-of-flight mass chamber was 10 times below that of the ionization chamber by differential pumping to avoid collision-induced fragmentation.

The spectrometer consisted of a repeller electrode, an extraction electrode, an einzel lens, electrostatic deflectors (horizontal, vertical), and a microchannel plate (MCP). A 500- $\mu$ m-wide slit was located on the extraction electrode perpendicular to the laser propagation direction (Fig. 2b) in order to collect the ions that were generated in the most tightly focused point of the laser beam (achieving ion collection from axially symmetric parallel beam geometry). The acceleration voltage was $4000 \mathrm{~V}$, and the extraction field potential $303 \mathrm{~V} \mathrm{~cm}^{-1}$ was optimized so that the double peaks of atomic ions that originated in the Coulomb explosion would have similar height. The resolution $(\mathrm{m} / \Delta m$, fwhm) was 560 at $m / z=129$. The output signal from the MCP (F4655-11X; Hamamatsu Photonics) was averaged by a digital oscilloscope (Wave Runner 6100, $1 \mathrm{GHz}$; LeCroy Japan) for 1000 shots. We observed only positive ions. The ion yield was obtained by integrating over the appropriate peaks in the time-of-flight spectrum. The error in the evaluation of the ion yield was approx. $\pm 5 \%$.

\section{Results and discussion}

\subsection{Mass spectra measured under a high laser intensity condition}

The mass spectrum (not shown) at around $10^{13} \mathrm{Wcm}^{-2}$ was dominated by molecular ions as in the case of butadiene [30]. In contrast, many atomic and fragment ions appeared in the mass spectrum in addition to intact molecular ions as the laser intensity increased to $10^{14} \mathrm{Wcm}^{-2}$. Fig. 3 shows the timeof-flight mass spectra of the four acetylene and two diacetylene derivatives at around $10^{14} \mathrm{Wcm}^{-2}$. The 
maximum charge state of the intact molecular ion was four for DIA and DIDA, and two for acetylene, DMA, DMDA, and IPA. In addition, we definitively observed the production of highly charged atomic ions such as $\mathrm{C}^{3+}$ and $\mathrm{I}^{5+}$. The mass spectrum measured at around $10^{14} \mathrm{Wcm}^{-2}$ is complicated, for the following reasons.

(1) Due to the spatial and temporal distribution of the laser intensity, higher charged states are formed at the most intense part of the laser pulse in both space and time. The lower charged states are formed at the wing of the laser beam (in space) as well as the leading and trailing edge of the laser pulse (in time) where the laser intensity is low. Therefore, the mass spectrum involves not only the atomic ions produced by the Coulomb explosion of multiply charged precursor ions which were generated at the stated intensity of $10^{14} \mathrm{Wcm}^{-2}$, but also the intact molecular ions and fragment ions of different origins [31]. We attempted to minimize this volume (space) effect by extracting the ions through a slit smaller than the Rayleigh length in this study [32]. The volume restriction along the laser beam propagation (perpendicular to the ion extraction direction) was successful; however, restriction of the volume along the ion extraction direction could not be achieved by the placement of a slit. Confinement of the exact ionization volume and the determination of the absolute ion yield [33] are difficult under our experimental conditions.

(2) Although the pulse width is short (40 fs), the successive absorption of photons by a molecular ion at the tailing edge of the laser pulse leads to heavy fragmentation by direct dissociation, predissociation, and dissociation in the vibrationally excited state [34].

(3) The atomic ions such as $\mathrm{H}^{+}, \mathrm{C}^{x+}(x=1-3), \mathrm{I}^{y+}(y=2-5)$, and some fragment ions showed double and triple peak structures. The origin of the multiple peak structure appears to be the Coulomb explosion (charge separation processes) of ions with certain kinetic energy. The ions with certain kinetic energy are emitted forward and backward against the ion flight axis, and therefore are detected with a certain time delay. The ions with nearly zero kinetic energy originating from the bond dissociation of a low charge state appeared in the middle of the multiple peaks. 
As clearly seen in Fig. 3a, acetylene showed simple fragment ion distributions. We observed atomic ions such as $\mathrm{H}^{+}, \mathrm{C}^{+}, \mathrm{C}^{2+}$, and $\mathrm{C}^{3+}$ as multiple peaks in addition to singly and doubly charged intact molecular ions. The results were identical to those reported by Cornaggia et al. [23]. The measured abundance ratio $(100: 2.20)$ between $\mathrm{m} / \mathrm{z}=13$ and 13.5 was identical to the expected isotope ratio of doubly charged molecular ions $(100: 2.19)$. Thus, the contribution of $\mathrm{CH}^{+}$to the peak at $\mathrm{m} / \mathrm{z}=$ 13 was negligible. In contrast, conventional electron impact ionization $(70 \mathrm{eV})$ produces $\mathrm{C}^{+}, \mathrm{CH}^{+}, \mathrm{C}_{2}^{+}$, and $\mathrm{C}_{2} \mathrm{H}^{+}$from acetylene [35]. A Coulomb explosion of acetylene induced by a collision with highenergy projectiles (e.g., $\mathrm{Ar}^{12+}$ ) also gives similar fragment ions as in the case of an electron impact study [36]. Unlike the case of acetylene, some fragment ions appeared in the mass spectra of DIA (Fig. 3b) and DIDA (Fig. 3d) in addition to atomic ions such as $\mathrm{C}^{x+}(x=1-3)$ and $\mathrm{I}^{y+}(y=1-6) \cdot \mathrm{C}_{2}^{+}, \mathrm{C}_{2} \mathrm{I}^{+}$, and $\mathrm{C}_{2} \mathrm{I}^{2+}$ were formed in the case of DIA, whereas $\mathrm{C}_{2}{ }^{+}, \mathrm{C}_{3}{ }^{+}, \mathrm{C}_{4}{ }^{+}, \mathrm{C}_{4} \mathrm{I}^{+}, \mathrm{C}_{4} \mathrm{I}^{2+}$, and $\mathrm{CI}^{+}$were formed in the case of DIDA. $\mathrm{C}_{2}^{+}$and $\mathrm{C}_{2} \mathrm{I}^{+}$are also formed by the electron impact ionization of DIA [37].

In a previous paper, we explained that the different fragment ion distributions between acetylene and DIA are due to the elongation of the chemical bond during higher-order ionization [24]. The nuclei have time to move apart from each other during the high-order ionization by femtosecond laser pulses, and terminal protons are expelled before reaching a higher charge state in the case of acetylene, while the timing of the $\mathrm{C}-\mathrm{I}$ bond elongation should be slower than that of the $\mathrm{C}-\mathrm{H}$ bond due to the large mass. Thus, some fragment ions remain in the mass spectrum of DIA as well as that of DIDA, even by the femtosecond laser ionization with infinite pulse duration. In contrast, neither $\mathrm{CI}^{+}$nor $\mathrm{C}_{2} \mathrm{I}^{+}$was observed in the case of IPA (Fig. 3f). Contrary to DIA, the mass spectrum of IPA indicates that the substitution of the terminal hydrogen of the acetylene moiety of phenyl acetylene by iodine does not alter the fragmentation processes of phenyl acetylene [6]. The dissociation of C-I bond may precede the dissociation of $\mathrm{C}_{6} \mathrm{H}_{5}-\mathrm{C}$ bond.

The mass spectra of DMA (Fig. 3c), DMDA (Fig. 3e), and IPA (Fig. 3f) consisted of $\mathrm{H}^{+}, \mathrm{H}_{2}^{+}, \mathrm{C}^{x^{+}}$ $(x=1-3)$ and a series of hydrocarbon fragment ions. These fragment ion distributions indicate that the dissociation of the carbon skeleton occurs as in the case of the electron impact study. Some fragment 
ions such as $\mathrm{C}_{m} \mathrm{H}_{n}^{+}(m=1-3, n=0-3)$ from DMA, $\mathrm{C}_{1} \mathrm{H}_{n}^{+}(n=1-3), \mathrm{C}_{2} \mathrm{H}_{n}^{+}(n=1-2), \mathrm{C}_{3} \mathrm{H}_{n}^{+}(n=0,1)$, $\mathrm{C}_{4} \mathrm{H}_{n}{ }^{+}(n=0,1)$, and $\mathrm{C}_{5} \mathrm{H}_{n}{ }^{+}(n=0,1,3)$ from DMDA, and $\mathrm{C}_{1} \mathrm{H}_{n}{ }^{+}(n=0-1)$ from IPA show a double or triple peak structure due to the Coulomb explosion. However, we leave these fragment ions unanalyzed because they should be formed at the low charge state of precursor ions, and thus they are not within the scope of this study.

\subsection{Decomposition of the angular distributions}

The ion yield depends strongly on the configuration between the direction of the laser polarization and the ion flight axis. Fig. 4 compares the angular distribution of carbon ions, $\mathrm{H}^{+}$, and $\mathrm{CH}_{3}{ }^{+}$ejected from the three acetylene and two diacetylene derivatives. Fig. 5 compares the angular distributions of iodine ions ejected from DIA and DIDA. As clearly seen in Figs. 4 and 5, atomic ions and $\mathrm{CH}_{3}{ }^{+}$showed anisotropic emission. This is because the detection of ions with certain kinetic energies is limited by the slit located on the extraction electrode in the time-of-flight mass spectrometer (Fig. 2b).

Although molecules have random orientations in the gas phase, energetic ions tend to be anisotropically emitted because molecules aligned to the laser electric fields have high ionization probability [38]. The geometric selection of molecules, namely, geometric alignment, is one of the most important features of tunneling ionization in strong laser fields [39]. The emission of ions is affected by the degree of geometric alignment by tunneling ionization, the molecular structure deformation before the Coulomb explosion, the rotational period of the precursor ions, and their kinetic energy. If the geometric alignment occurs in a very strict manner, the angular distribution becomes very sharp. As was seen in the sharp angular distribution of ions (Fig. 4a), the geometric alignment was successful in the case of acetylene. The geometric alignment depends on the configuration of the highest occupied molecular orbital (HOMO) [40]. For the cases of other acetylene derivatives, the HOMOs have similar axial symmetry [41]. Therefore, the degree of geometric alignment of these molecules is expected to be similar to that of acetylene. 
Molecular deformation, especially in a bending coordinate, results in an angular distribution that is different from that expected for a neutral molecule [16]. However, the anisotropy of ion emission by a Coulomb explosion would be reduced if the dissociation rate is comparable to and/or slower than the rotational period. Molecular ions as well as ions without kinetic energy are detected in the same abundance under both the parallel and orthogonal conditions because they have nearly zero kinetic energy, and thus such ions can pass through a narrow slit located on the extraction electrode in the mass spectrometer. Thus their emission is referred to as "isotropic."

To facilitate the discussion of the ion emission results, we tried to extract quantitative information about the direction of ion ejection. For the sake of simplicity, we assumed that the ions were ejected in four different manners: parallel $(\theta=0,180)$, orthogonal $(\theta=90,270)$, diagonal $(\theta=45,135,225,315)$, and isotropic relative to the laser polarization direction, as shown in Fig. 6a. The angle measured with respect to the laser polarization direction from the ion flight axis to the detector was defined as $\theta$ (Fig. 2b). The ion yield $(y)$ was fitted by Eq. (1) with four coefficients: $a$ (parallel), $b$ (diagonal), $c$ (orthogonal), and $d$ (isotropic). The sum of these coefficients was normalized to unity.

$$
y=a \cos ^{4}\left(\frac{\pi}{180} \theta\right)+b\left[\cos ^{4}\left(\frac{\pi}{180}(\theta+45)\right)+\cos ^{4}\left(\frac{\pi}{180}(\theta-45)\right)\right]+c \cos ^{4}\left(\frac{\pi}{180}(\theta-90)\right)+d
$$

The trajectory of atoms $(\mathrm{H}, \mathrm{I})$ and substituents $\left(\mathrm{CH}_{3}\right)$ located on the edge of the molecular frame is not hindered by other atoms, and therefore it is expected that they are ejected in a parallel direction. In contrast, the carbon ion emission should be affected by terminal substituents as well as the deformation of the molecular structure, the original location of the carbon, the molecular length, and the molecular rotation. Therefore, we will first discuss the ejection of $\mathrm{H}^{+}$, iodine ions, and $\mathrm{CH}_{3}{ }^{+}$, and then carbon ions.

\subsection{The angular distribution of terminal substituents: $\mathrm{H}^{+}$, iodine ions, and $\mathrm{CH}_{3}{ }^{+}$}

The angular distribution of $\mathrm{H}^{+}$ejected from acetylene was very narrow along the laser polarization direction. Though it was not well fitted by the cosine functions to the fourth power but was well fitted by the cosine functions to the 8 th power, only the parallel $(89.2 \%)$ and isotropic $(10.8 \%)$ components 
were extracted. The angular distribution of $\mathrm{H}^{+}$was identical for DMA and DMDA. The contribution of the orthogonal component was negligible; therefore, it was assumed to be zero in those cases. The angular distribution of $\mathrm{H}^{+}$was decomposed to parallel (DMA, 59.3\%; DMDA, 55.9\%), diagonal (DMA, 20.1\%; DMDA, 13.7\%), and isotropic components (DMA, 20.6\%; DMDA, 30.4\%). The broad angular distribution of $\mathrm{H}^{+}$that originated from DMA and DMDA can be explained in terms of their hybridizations of $\mathrm{C}-\mathrm{H}$ bonds $\left(\mathrm{sp}^{3}\right)$. DMA has $\mathrm{D}_{3 \mathrm{~d}}$ symmetry, and the $\mathrm{C}-\mathrm{H}$ bond axis in the methyl group is not parallel to the triple bond axis; thus, a Coulomb explosion of the proton has a certain momentum orthogonal to the triple-bond axis even when the geometric alignment takes place. Based on the similar contribution of each component to the angular distributions of DMA and DMDA, we suggest that the ejection of protons is independent of the length of molecules.

Iodine ions (expect $\mathrm{I}^{+}$) were predominantly ejected along the laser polarization direction, and the distribution became narrower as the charge state increased. Fig. 6b,c shows the extracted coefficients as a function of the iodine charge number. The contribution of the orthogonal component was negligible, and thus it was assumed to be zero to have a best fit. As the charge number increased, the contribution of the parallel component increased and that of the diagonal component decreased. The isotropic component was only found in $\mathrm{I}^{+}$. We note that the angular distributions of multiply charged iodine ions cannot be fit by using an isotropic component instead of a diagonal component. The trend of each contribution as a function of the charge number was similar between DIA and DIDA, except for I ${ }^{+}$. We thus concluded that the angular distribution of the iodine ions other than $\mathrm{I}^{+}$is not significantly dependent on the length of the molecules.

Because a methyl group is located on the edge of the molecular frame, $\mathrm{CH}_{3}{ }^{+}$was expected to be ejected along the molecular axis and thus in a parallel direction. However, the distribution of $\mathrm{CH}_{3}{ }^{+}$was broader than that of the proton. Fig. 7a shows the coefficients of $\mathrm{CH}_{3}{ }^{+}$that originated from DMA and DMDA. The orthogonal component was negligibly small, and it was therefore assumed to be zero to reduce the number of the fitting parameters. The contribution of each component to the angular distribution of $\mathrm{CH}_{3}^{+}$was parallel (DMA, 37.9\%; DMDA, 28.8\%), diagonal (DMA, 0\%; DMDA, 6.5\%) 
and isotropic (DMA, 62.0\%; DMDA, 64.7\%). The parallel component in the $\mathrm{CH}_{3}{ }^{+}$angular distribution of DMA was slightly higher than that of DMDA. However, the major ( $>60 \%)$ isotropic component was the same.

It should be noted that the origin of $\mathrm{CH}_{3}{ }^{+}$is not a singly charged molecular ion but doubly and perhaps higher charge states, because the electron stays with the fragment of higher ionization energy when the dissociation occurs. For example, photoirradiation to $\mathrm{DMDA}^{+}$gives not $\mathrm{CH}_{3}{ }^{+}$but $_{5} \mathrm{H}_{3}{ }^{+}$[42] . In contrast, the charge separation of $\mathrm{DMDA}^{2+}$ forms $\mathrm{CH}_{3}{ }^{+}$[43]. It was expected that the ejection of $\mathrm{CH}_{3}{ }^{+}$occurs at a charge number higher than two. However, the rate of $\mathrm{CH}_{3}{ }^{+}$ejection should be slow and comparable to the time scale of molecular rotation, judging from the dominance of the isotropic component in angular distribution. We therefore calculated the rotational period, $T_{\text {rot }}=\frac{h}{2 B}$, where $h$ and $B$ are Planck's constant and the rotational constant, respectively. The rotational constants of acetylene, DMA, and DMDA are $1.17[44], 1.12 \times 10^{-1}$, and $3.72 \times 10^{-2} \mathrm{~cm}^{-1}$ [45], respectively. Therefore, the rotational periods of acetylene, DMA, and DMDA were calculated to be 14.2, 148, and 447 ps, respectively. Although it is difficult to show the correlations among the angular distribution, dissociation rate and rotational period of precursor ions, the formation of $\mathrm{CH}_{3}{ }^{+}$may be on the order of hundreds of picoseconds.

We concluded that the angular distribution of the terminal atoms other than $\mathrm{I}^{+}$showed a predominant ejection along the laser polarization direction in all of the molecules examined. In contrast, terminal $\mathrm{CH}_{3}{ }^{+}$was ejected mostly $(>60 \%)$ in an isotropic manner because the low charge states of molecule rotates to some extent before the $\mathrm{CH}_{3}{ }^{+}$ejection. That is, the molecular rotation diminishes the effect of geometric alignment by tunneling ionization. The emission of $\mathrm{I}^{+}$is also considered to be a slow-enough process to be affected by molecular rotation.

\subsection{The angular distributions of carbon ions ejected from acetylene, DMA and DMDA}


The angular distributions of carbon ions were dependent on their charge number and the length of the molecule. Fig. 7a shows the coefficients derived from the angular distribution of carbon ions ejected from acetylene, DMA, and DMDA. The carbon ions were ejected from acetylene in a parallel direction. The $\mathrm{C}^{+}$ejection from acetylene consisted of $87.8 \%$ parallel, $0 \%$ diagonal, and $12.2 \%$ isotropic components. The orthogonal component was not considered. It should be mentioned that we could fit the data of $\mathrm{C}^{2+}$ and $\mathrm{C}^{3+}$ from acetylene by the cosine functions to the fourth power but we could fit the data well by the cosine functions to the 8th power. Although the reason for the narrow distribution is not certain, we show the evaluated coefficients of $\mathrm{C}^{2+}$ and $\mathrm{C}^{3+}$ in Fig. 7a because only the parallel component was extracted.

The angular distributions of carbon ions expelled from DMA and DMDA were broader than that from acetylene. The coefficients were dependent on the carbon charge, and the length of the molecules (Fig. 7a). The angular distributions of $\mathrm{C}^{+}, \mathrm{C}^{2+}$ and $\mathrm{C}^{3+}$ ejected from DMA and DMDA were decomposed into parallel, diagonal, and isotropic components. The orthogonal component was negligibly small, and thus it was assumed to be zero to reduce the ambiguity of the other coefficients. The contributions of each component to the angular distribution of DMA were parallel $\left(\mathrm{C}^{+}, 24.1 \% ; \mathrm{C}^{2+}, 49.3 \% ; \mathrm{C}^{3+}, 75.8 \%\right)$, diagonal $\left(\mathrm{C}^{+}, 6.9 \% ; \mathrm{C}^{2+}, 12.7 \% ; \mathrm{C}^{3+}, 0 \%\right)$ and isotropic $\left(\mathrm{C}^{+}, 68.9 \% ; \mathrm{C}^{2+}, 38.0 \% ; \mathrm{C}^{3+}, 24.2 \%\right)$. Those of DMDA were parallel $\left(\mathrm{C}^{+}, 6.5 \%\right.$; $\mathrm{C}^{2+}, 29.1 \%$; $\left.\mathrm{C}^{3+}, 60.3 \%\right)$, diagonal $\left(\mathrm{C}^{+}, 0 \% ; \mathrm{C}^{2+}, 13.1 \% ; \mathrm{C}^{3+}, 15.0 \%\right)$ and isotropic $\left(\mathrm{C}^{+}, 93.5 \% ; \mathrm{C}^{2+}, 57.8 \% ; \mathrm{C}^{3+}, 24.7 \%\right)$. As the charge number increased, the distribution of carbon ions became narrower; in other words, the contribution of the parallel component became dominant whereas the isotropic component decreased. This trend was observed in both DMA and DMDA. As the length of the molecules became longer, the angular distribution of carbon ions became broader; that is, the contribution of the parallel component decreased and that of the isotropic component increased.

The origin of the isotropic component — the key to solve the length effect—in the angular distribution of carbon ions needs explanation. It is necessary to consider that we observe the ions of different origins simultaneously due to the spatial and temporal distribution of laser intensity, as 
mentioned in Section 3.1 above. The charge of the precursor ion of $\mathrm{C}^{+}, \mathrm{C}^{2+}$ and $\mathrm{C}^{3+}$ is different because the ionization potential of $\mathrm{C}, \mathrm{C}^{+}$and $\mathrm{C}^{2+}$ are $11.3,24.4$, and $47.9 \mathrm{eV}$, respectively. It is expected that $\mathrm{C}^{3+}$ is formed at the most intense central part (the stated intensity of $10^{14} \mathrm{Wcm}^{-2}$ ) of the laser beam, whereas $\mathrm{C}^{+}$is formed at the wing of the laser beam (in space) as well as the leading and trailing edge of the laser pulse (in time) where the laser intensity is low.

The emission process of $\mathrm{C}^{2+}$ and $\mathrm{C}^{3+}$ is rather straightforward. As the angular distribution of $\mathrm{C}^{2+}$ and $\mathrm{C}^{3+}$ was aligned to the laser polarization direction, the structure of the highly charged precursor ion, the origin of $\mathrm{C}^{2+}$ and $\mathrm{C}^{3+}$, is expected to be linear. The geometry of highly charged, at least $6+$, methylacetylene $\left(\mathrm{CH}_{3}-\mathrm{C} \equiv \mathrm{C}-\mathrm{H}\right)$ before the Coulomb explosion has been investigated by covariance mapping techniques at $5 \times 10^{15} \mathrm{Wcm}^{-2}$ [46]. Three carbons have an elongated $(2.1$ times compared to that of the cation radical) but still linear geometry at $5 \times 10^{15} \mathrm{Wcm}^{-2}$. The Coulomb repulsion between the carbon ions in such highly charged states should be strong, and thus the ion emission is an instantaneous process. At the same time, the terminal carbon would be apart from the molecular frame by the deformation in stretching coordinates at highly charged states, and thus the terminal carbon would not inhibit the ejection of carbon participating in the triple bond. By analogy to the investigations of methylacetylene [46], it is expected that the linear but elongated carbon geometry of the precursor ion of $\mathrm{C}^{2+}$ and $\mathrm{C}^{3+}$ is maintained before the Coulomb explosion in the cases of DMA and DMDA.

Contrary to the emission of $\mathrm{C}^{2+}$ and $\mathrm{C}^{3+}$, the time scale of $\mathrm{C}^{+}$emission should be slow due to the weak Coulomb repulsion. Assuming that the time scale of $\mathrm{C}^{+}$emission and molecular rotation is comparable, the emission process is strongly affected not only by the molecular rotation, but also by the deformation of the molecules and the original location of the carbon ions. These factors are strongly related to the length of the molecule. The emission of $\mathrm{C}^{+}$from DMDA was more isotropic (94\%) than that of $\mathrm{CH}_{3}{ }^{+}(65 \%)$. In contrast, those from DMA were similar: $69 \%\left(\mathrm{C}^{+}\right)$and $62 \%\left(\mathrm{CH}_{3}{ }^{+}\right)$.

Based on the similarity of isotropic and parallel components, the emission of $\mathrm{C}^{+}$and $\mathrm{CH}_{3}{ }^{+}$from DMA should be affected by molecular rotation in the similar way. In contrast, the different angular distributions between $\mathrm{C}^{+}$and $\mathrm{CH}_{3}{ }^{+}$from DMDA cannot be explained in terms of molecular rotation 
alone, because the rotational period of DMDA is longer than that of DMA. We thus need to consider the original location of the carbons. Carbons in DMA were ejected from two different sites, namely $\mathrm{C}_{\mathrm{A}}$ and $\mathrm{C}_{\mathrm{B}}\left(\mathrm{C}_{\mathrm{A}} \mathrm{H}_{3}-\mathrm{C}_{\mathrm{B}}=\mathrm{C}_{\mathrm{B}}-\mathrm{C}_{\mathrm{A}} \mathrm{H}_{3}\right)$. Carbons were ejected from three different sites from DMDA, namely $\mathrm{C}_{\mathrm{A}}, \mathrm{C}_{\mathrm{B}}$, and $\mathrm{C}_{\mathrm{C}}\left(\mathrm{C}_{\mathrm{A}} \mathrm{H}_{3}-\mathrm{C}_{\mathrm{B}} \equiv \mathrm{C}_{\mathrm{C}}-\mathrm{C}_{\mathrm{C}} \equiv \mathrm{C}_{\mathrm{B}}-\mathrm{C}_{\mathrm{A}} \mathrm{H}_{3}\right)$. It may be reasonable to assume that carbons $\left(\mathrm{C}_{\mathrm{A}}\right)$ located on the edge of the molecular frame show an angular distribution similar to that of $\mathrm{CH}_{3}{ }^{+}$. In contrast, once the molecular rotation and elongation of $\mathrm{C}-\mathrm{C}$ bonds occurs simultaneously, the emission of $\mathrm{C}_{\mathrm{B}}{ }^{+}$and $\mathrm{C}_{\mathrm{C}}{ }^{+}$ should proceed in an isotropic manner. The influence of molecular rotation and the original location of carbon atom should be especially significant for the emission of $\mathrm{C}_{\mathrm{C}}$ from DMDA. We conclude that the molecular length effect on the carbon ion emission is explained in terms of the molecular rotation, the deformation of the molecule in the stretching coordinates and the original location of the carbon atoms.

\subsection{The angular distribution of the carbon ions ejected from DIA and DIDA}

The angular distributions of carbon ions ejected from DIA and DIA showed the opposite trends compared to that from DMA and DMDA: they were in the orthogonal direction rather than the parallel direction, and they became narrower as the length of the molecule became longer. The angular distributions of $\mathrm{C}^{+}$and $\mathrm{C}^{2+}$ ejected from DIA and DIDA were decomposed into parallel, diagonal, orthogonal, and isotropic components. The parallel component was zero. The best fit results are shown in Fig. 7b. The contribution of each component to the angular distribution of DIA was diagonal $\left(\mathrm{C}^{+}\right.$, $\left.19.7 \% ; C^{2+}, 36.1 \%\right)$, orthogonal $\left(C^{+}, 30.5 \% ; C^{2+}, 45.6 \%\right)$ and isotropic $\left(C^{+}, 48.8 \% ; C^{2+}, 18.1 \%\right)$. Those

of DIDA were diagonal $\left(\mathrm{C}^{+}, 35.9 \% ; \mathrm{C}^{2+}, 34.1 \%\right)$, orthogonal $\left(\mathrm{C}^{+}, 40.9 \% ; \mathrm{C}^{2+}, 65.9 \%\right)$ and isotropic $\left(\mathrm{C}^{+}\right.$, $\left.22.7 \% ; \mathrm{C}^{2+}, 0 \%\right)$

We noted that the ejection of $\mathrm{C}^{2+}$ from DIDA was focused to orthogonal directions $(66 \%)$. The angular distribution became narrower along the orthogonal direction as the charge of carbon increased in the cases of DIA and DIDA (Figs. 4, 7b). In contrast, it became narrower along the parallel direction as the charge of the carbon ions increased in the cases of DMA and DMDA (Figs. 4, 7a). More interestingly, the angular distribution of carbon ejected from DIA and DIDA became sharp as the length 
of the molecule became longer. This trend was the opposite of that from DMA and DMDA. That is, the isotropic component decreased in the diiodoacetylenes (DIA, DIDA) but increased in the dimethylacetylenes (DMA, DMDA) as the length of the molecule became longer. These results are quite informative because the terminal substituent effect is more significant in longer iodine-substituted acetylenes although the distance between terminal iodine atoms is long.

In order to explain the predominant carbon ion emission in the orthogonal direction (especially in the case of DIDA), we need to consider the deformation of the molecular skeleton in bending coordinates, the deformation of the molecular skeleton in stretching coordinates, and the effects of molecular rotation on the geometric alignment conditions.

First, the deformation of the molecular skeleton in bending coordinates is necessary to emit carbon ions in the orthogonal direction. Judging from the angular distribution of carbon ions, acetylene maintains the $\mathrm{D}_{\infty \mathrm{h}}$ symmetry before a Coulomb explosion. Assuming that DIA maintains the $\mathrm{D}_{\infty \mathrm{h}}$ symmetry before a Coulomb explosion, the angular distribution should be similar to that of acetylene, because the ions generated within the framework of DIA would not have a certain momentum orthogonal to the molecular axis. Therefore, the ejection of carbon ions orthogonal to the laser polarization direction requires a certain perturbation of the molecular structure before the Coulomb explosion. The symmetry of DIA may become different by the deformation of the molecular skeleton in the bending coordinates; e.g., developing the symmetry of $\mathrm{C}_{2 \mathrm{~h}}$. The deformation would be triggered by stripping $\pi$ electrons from the acetylene moiety that loosens the rigid character of the triple bond (sp), and gains a single-bond-like $\left(\mathrm{sp}^{3}\right)$ character.

Second, the deformation in stretching coordinates should be minimal before a Coulomb explosion because ions fly in the parallel directions if the significant bond elongation occurs in advance. For example, the hydrogen in acetylene disappears immediately as a proton by means of Coulomb repulsion, and thus the carbons do not fly to the orthogonal direction. Although it is not clear whether the terminal $\mathrm{CH}_{3}$ can block the ejection of other carbon atoms judging from the mass spectra alone, the relatively 
light and fragmentable terminal substituent $\mathrm{CH}_{3}$ does not have influence on the carbon ion emissions in the cases of DMA and DMDA.

In contrast, the persistent presence of iodine atoms surrounding the carbon atoms allows the molecular frame distortion in bending coordinates prior to bond elongation. We conclude that the molecular deformation in bending coordinates is assisted by the persistent presence of iodine in the cases of DIA and DIDA under the influence of strong laser fields. For the case of DIDA, the bending effect may be more important to have a sharp angular distribution of carbon ions along the orthogonal direction. As the angular distribution of iodine ions between DIA $\left(I-\mathrm{C}_{\mathrm{B}}=\mathrm{C}_{\mathrm{B}}-\mathrm{I}\right)$ and DIDA $\left(\mathrm{I}-\mathrm{C}_{\mathrm{B}} \equiv \mathrm{C}_{\mathrm{C}^{-}}\right.$ $\left.\mathrm{C}_{\mathrm{C}} \equiv \mathrm{C}_{\mathrm{B}}-\mathrm{I}\right)$ is similar, we could expect a similar degree of inhibition of $\mathrm{C}_{\mathrm{B}}$ ion emission by the adjacent iodine. If the molecular frame distortion of DIDA occurs along the bending coordinates, carbons $\left(\mathrm{C}_{\mathrm{C}}\right)$ that are connected by a single bond and located in the central part of the molecule would be strongly affected, while the displacement of carbon $\left(\mathrm{C}_{\mathrm{B}}\right)$ next to iodine may relatively be small and similar to that of DIA.

Third, the geometric alignment situation is strictly maintained before Coulomb explosion because molecular rotation is frozen (especially in the case of DIDA). We calculated the rotational constant and period from the corresponding bond lengths and atom masses. The rotational constants of DIA and

DIDA as rigid rotor were calculated to be $9.89 \times 10^{-3}$ and $4.33 \times 10^{-3} \mathrm{~cm}^{-1}$, respectively. The rotational periods of DIA and DIDA were thus calculated to be 1.68 and 3.84 ns, respectively. These findings indicate that the rotations of DIA and DIDA are each almost frozen compared to those of the other molecules examined.

\section{Conclusion}

We investigated the Coulomb explosion of atomic ions and $\mathrm{CH}_{3}{ }^{+}$for a series of acetylene derivatives in intense femtosecond laser fields. The substitution of iodines provided the distinct angular distribution of carbon ions: the emission direction with respect to the laser polarization direction, and the dependence on the molecular length. The origins of the differences between the iodine-substituted acetylenes and the 
methyl-substituted acetylenes are explained in terms of the presence of iodines in close proximity to carbons even at highly charged states. These differences are due, in other words, to the different time scales of the departures of the terminal atoms (substituents), the different degrees of the blocking of carbon ion emission, and the different degrees of the deformation of the molecular skeleton in bending and/or stretching coordinates.

In addition, the molecular rotation is almost frozen in the cases of DIA and DIDA, and thus the geometric alignment condition is maintained and the deformation effect is emphasized. A recent experiment examining a collision with a high-energy projectile also showed that the carbon ions in diiodoacetylene are ejected perpendicularly with respect to the iodine ions [25]. As the instantaneous generation $(<1$ fs) of highly charged states was achieved in the collision experiments, carbon ions generated within the framework of neutral DIA would not have a certain momentum orthogonal to the molecular axis. However, it is not certain that the ion emissions are affected by the deformation of the molecular structure in the collision experiments. In contrast, the nuclei have time to move apart from each other during the femtosecond laser ionization. We are now conducting a systematic study of the ionization and Coulomb explosion dynamics for a series of diiodoalkanes, which have bended carbon chains. The correlation between the molecular structure (i.e., the terminal atoms and the length of the molecule) and the deformation of the molecular skeleton (bending and stretching) prior to a Coulomb explosion will also be investigated.

\section{Acknowledgments}

The present research was partially supported by JST PRESTO program and JSPS KAKENHI Grant Number 26620014. 


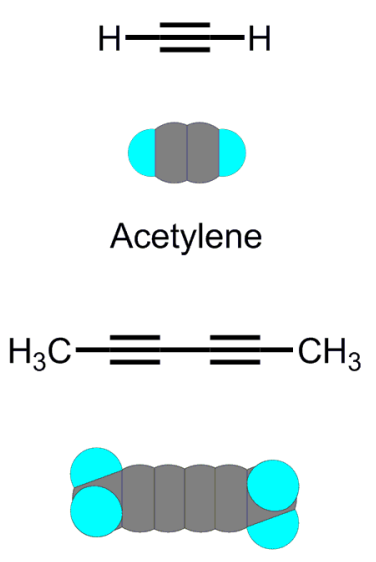

DMDA
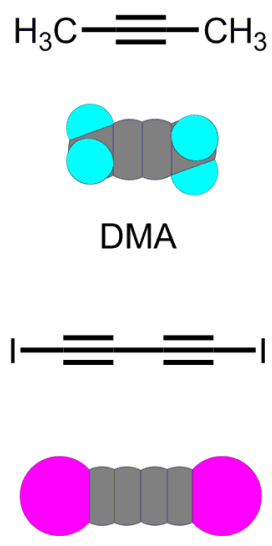

DIDA
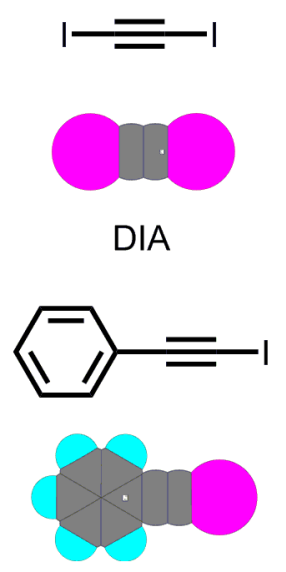

IPA

Fig. 1. The molecular structures of the acetylene derivatives examined. 
a)
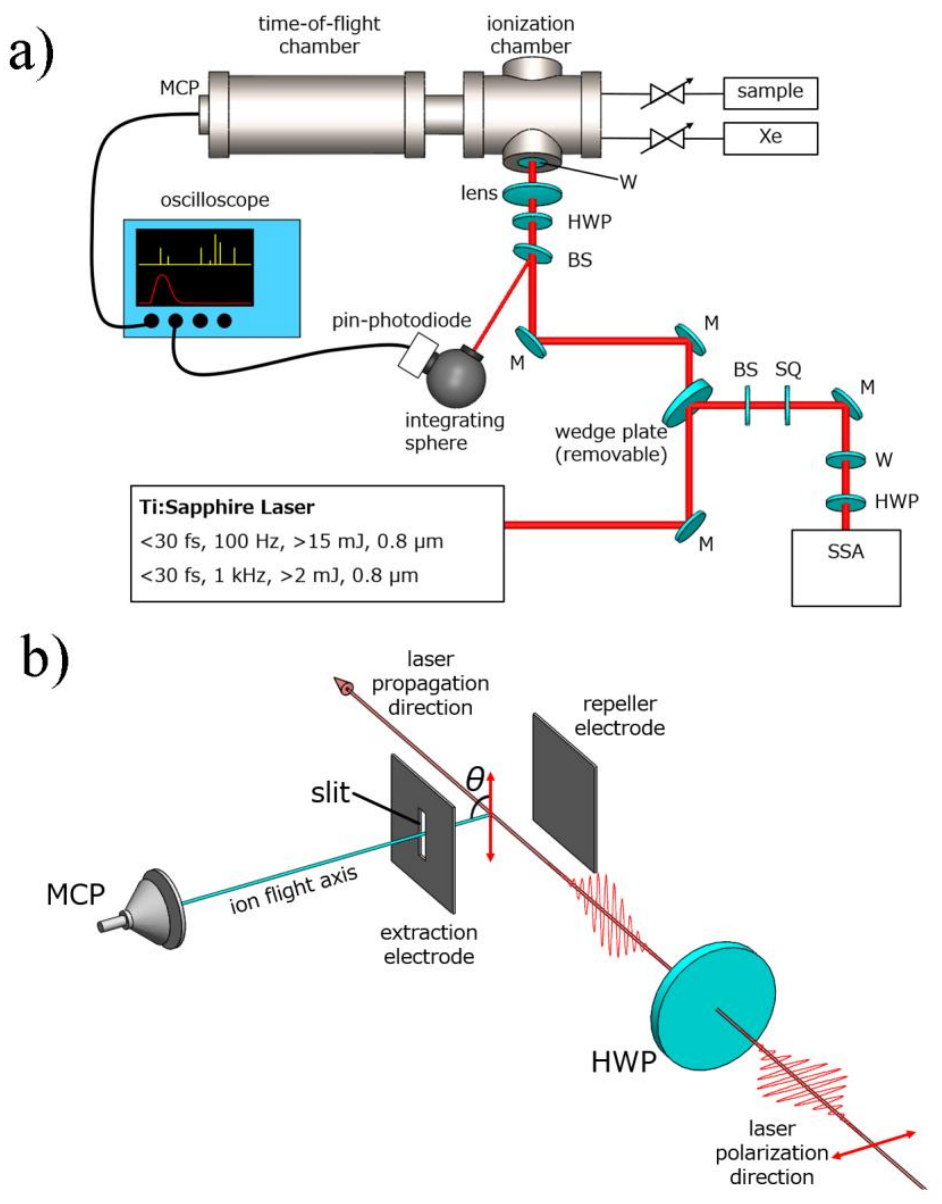

Fig. 2. Schematic of the (a) experimental set-up, (b) the configuration between the ion flight axis and the laser polarization direction. The angle $\theta$ measured with respect to the laser polarization direction from the ion flight axis to the detector. SSA, second-order single-shot autocorrelator; M, dielectric mirror; BS, anti-reflection coated beam splitter; HWP, zero-order half-wave plate; SQ, quartz plate; W, ionization chamber window. 


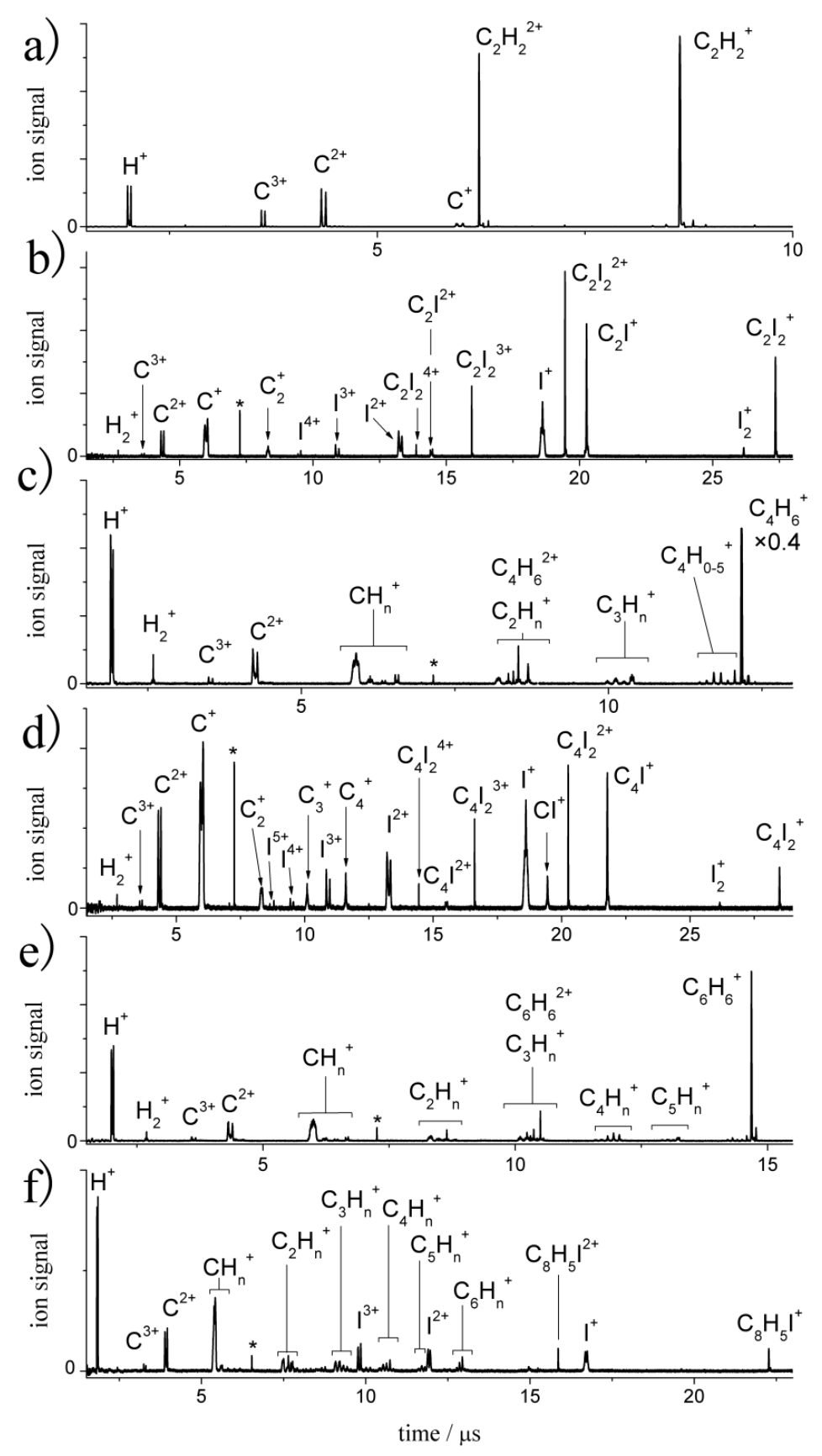

Fig. 3. Time-of-flight mass spectra of (a) acetylene at $5.2 \times 10^{14} \mathrm{Wcm}^{-2}$, (b) diiodoacetylene (DIA) at $3.6 \times 10^{14} \mathrm{Wcm}^{-2}$, (c) 2-butyne (DMA) at $4.9 \times 10^{14} \mathrm{Wcm}^{-2}$, (d) diiododiacetylene (DIDA) at $4.0 \times 10^{14}$ $\mathrm{Wcm}^{-2}$, (e) 2, 4-hexadiyne (DMDA) at $3.5 \times 10^{14} \mathrm{Wcm}^{-2}$, and (f) 2-iodo-1-phenylacetylene (IPA) at $3.9 \times 10^{14} \mathrm{Wcm}^{-2}$. The laser polarization was parallel (a, c, e, f) or orthogonal $(\mathrm{b}, \mathrm{d})$ to the ion flight axis. The spectrum $\mathrm{f}$ was taken under the different spectrometer condition (The acceleration voltage was 5000 $\mathrm{V}$, and the extraction field potential $472 \mathrm{~V} \mathrm{~cm}^{-1}$ ). $*$ Contaminated water. A part of the spectra a and $\mathrm{b}$ was taken from the literature [24]. Copyright Wiley-VCH Verlag. Reproduced with permission. 

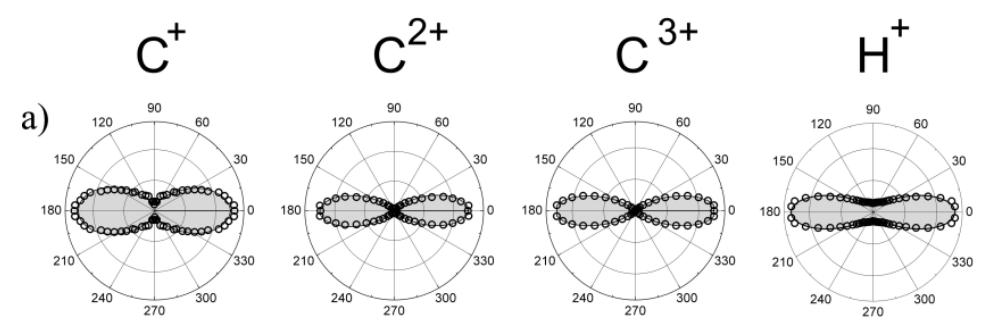

\section{$\mathrm{CH}_{3}^{+}$}

b)
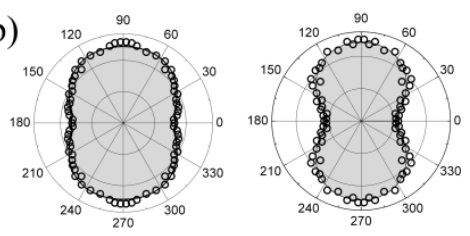

c)
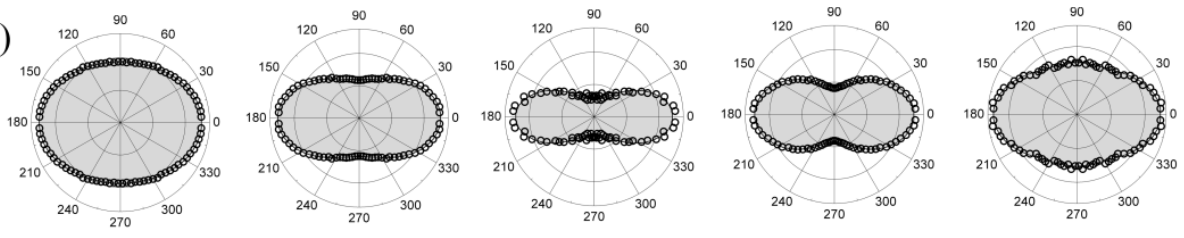

d)

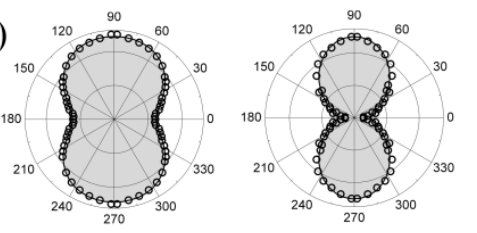

e)
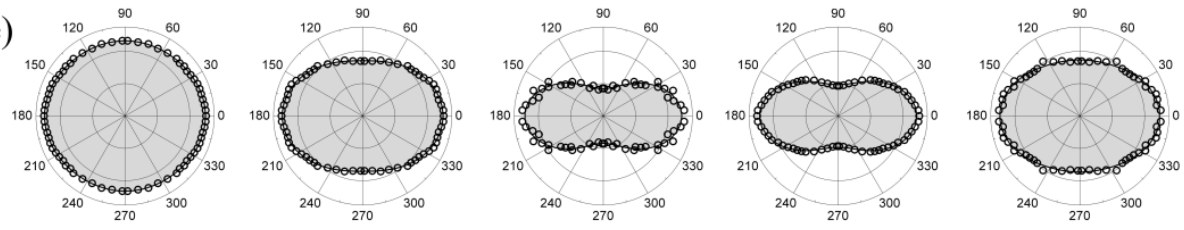

Fig. 4. Angular distributions of $\mathrm{C}^{+}, \mathrm{C}^{2+}, \mathrm{C}^{3+}, \mathrm{H}^{+}$, and $\mathrm{CH}_{3}{ }^{+}$ejected from (a) acetylene at $5.2 \times 10^{14}$ $\mathrm{Wcm}^{-2}$, (b) diiodoacetylene (DIA) at $4.4 \times 10^{14} \mathrm{Wcm}^{-2}$, (c) 2-butyne (DMA) at $4.9 \times 10^{14} \mathrm{Wcm}^{-2}$, (d) diiododiacetylene (DIDA) at $3.0 \times 10^{14} \mathrm{Wcm}^{-2}$, and (e) 2, 4-hexadiyne (DMDA) at $3.5 \times 10^{14} \mathrm{Wcm}^{-2}$. The plot of $\mathrm{C}^{3+}$ (DIA, DIDA) was not obtained due to the low signal-to-noise ratio. The angles measured with respect to the polarization plane of the laser fields from the ion flight axis to the detector were as follows: parallel $\left(0^{\circ}, 180^{\circ}\right)$, and orthogonal $\left(90^{\circ}, 270^{\circ}\right)$. The radius represents the relative ion intensity. The solid lines are best fitted by the combination of cosine functions to the fourth power. The data of $\mathrm{C}^{2+}, \mathrm{C}^{3+}$, and $\mathrm{H}^{+}$from acetylene were fitted by the cosine functions to the 8 th power. The area enclosed by solid lines is fitted by Eq. (1). A part of each of the spectra a and b was taken from the literature [24]. Copyright Wiley-VCH Verlag. Reproduced with permission. 
a)
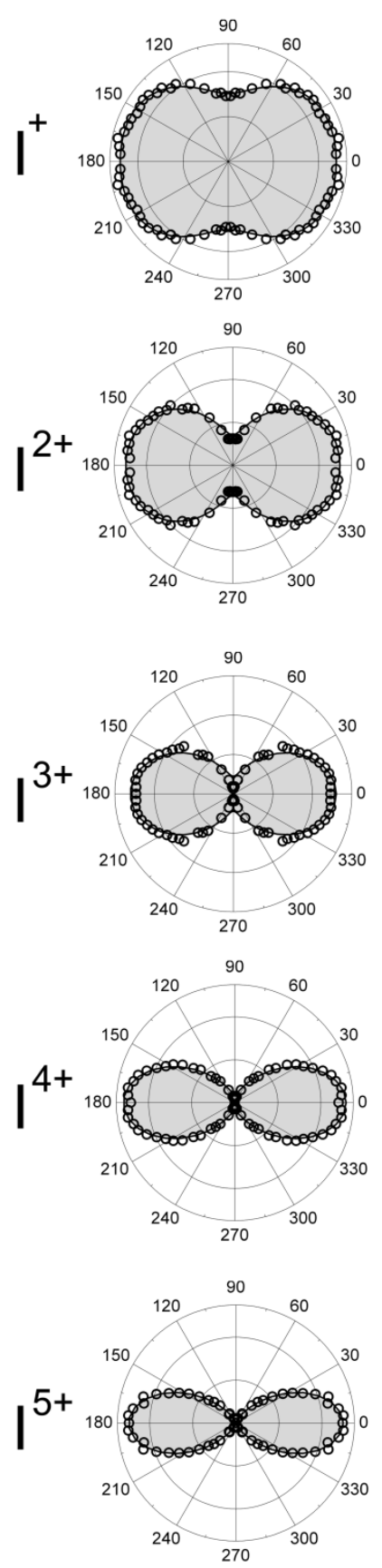

b)
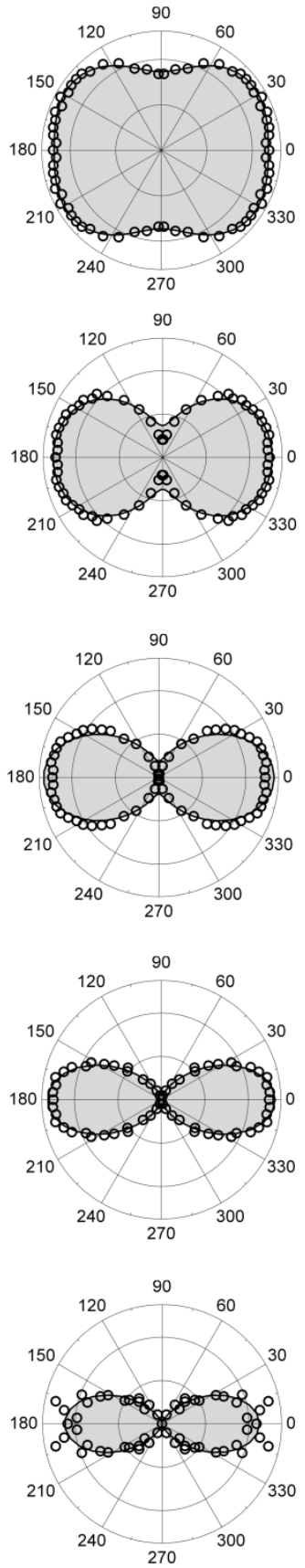

Fig. 5. Angular distributions of iodine ions ejected from (a) diiodoacetylene (DIA) at $4.4 \times 10^{14} \mathrm{Wcm}^{-2}$, and (b) diiododiacetylene (DIDA) at $3.0 \times 10^{14} \mathrm{Wcm}^{-2}$. The angles measured with respect to the polarization plane of the laser fields from the ion flight axis to the detector were as follows: parallel $\left(0^{\circ}\right.$, $\left.180^{\circ}\right)$, and orthogonal $\left(90^{\circ}, 270^{\circ}\right)$. The radius represents the relative ion intensity. The solid lines are best fitted by the combination of cosine functions to the fourth power. The area enclosed by solid lines is fitted by Eq. (1). 

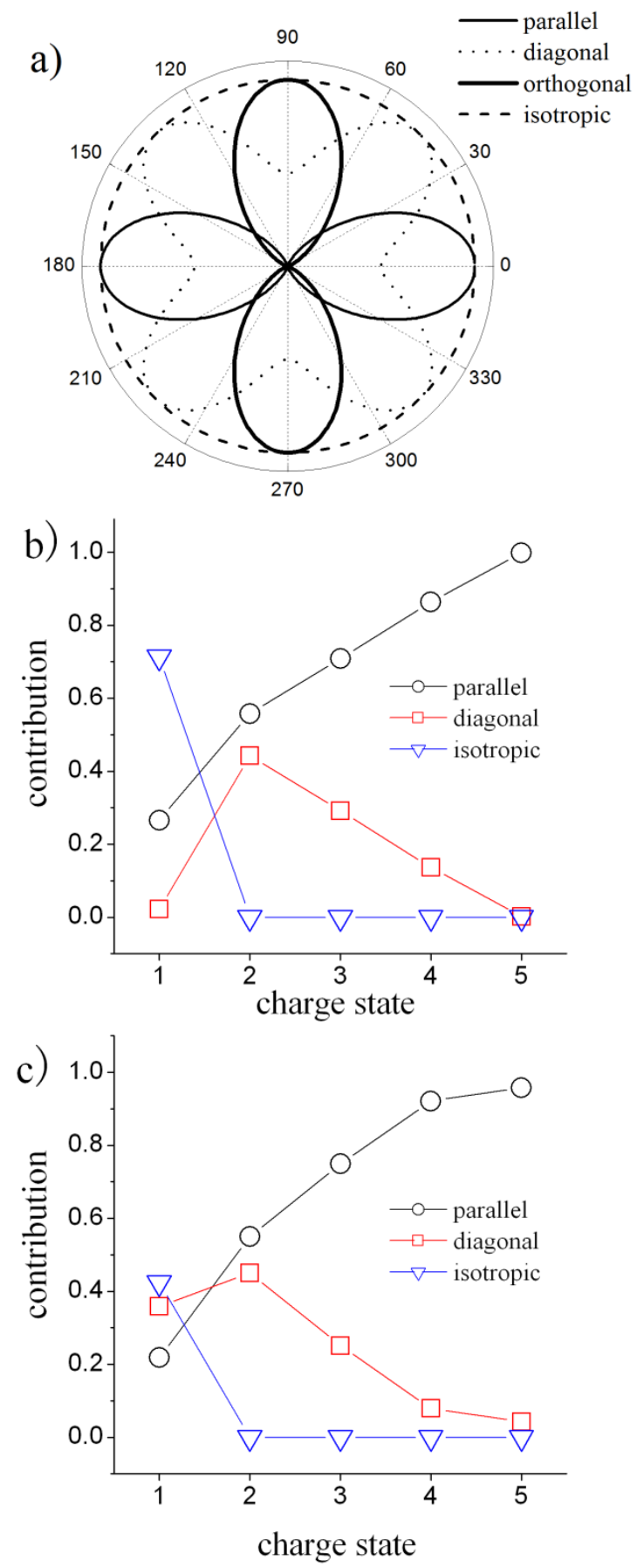

Fig. 6. (a) Angular distribution of the parallel (solid line), diagonal (dotted line), orthogonal (bold line), and isotropic (broken line) components used in Eq. (1). The contribution of iodine ions ejected parallel (black open circles), diagonal (red open squares), and isotropic (inverted blue open triangles) to the laser polarization direction in the cases of (b) diiodoacetylene (DIA) at $4.4 \times 10^{14} \mathrm{Wcm}^{-2}$, and (c) diiododiacetylene (DIDA) at $3.0 \times 10^{14} \mathrm{Wcm}^{-2}$. The angular distributions of iodine ions shown in Fig. 5 were fitted by Eq. (1). The solid lines are to guide the eyes. 

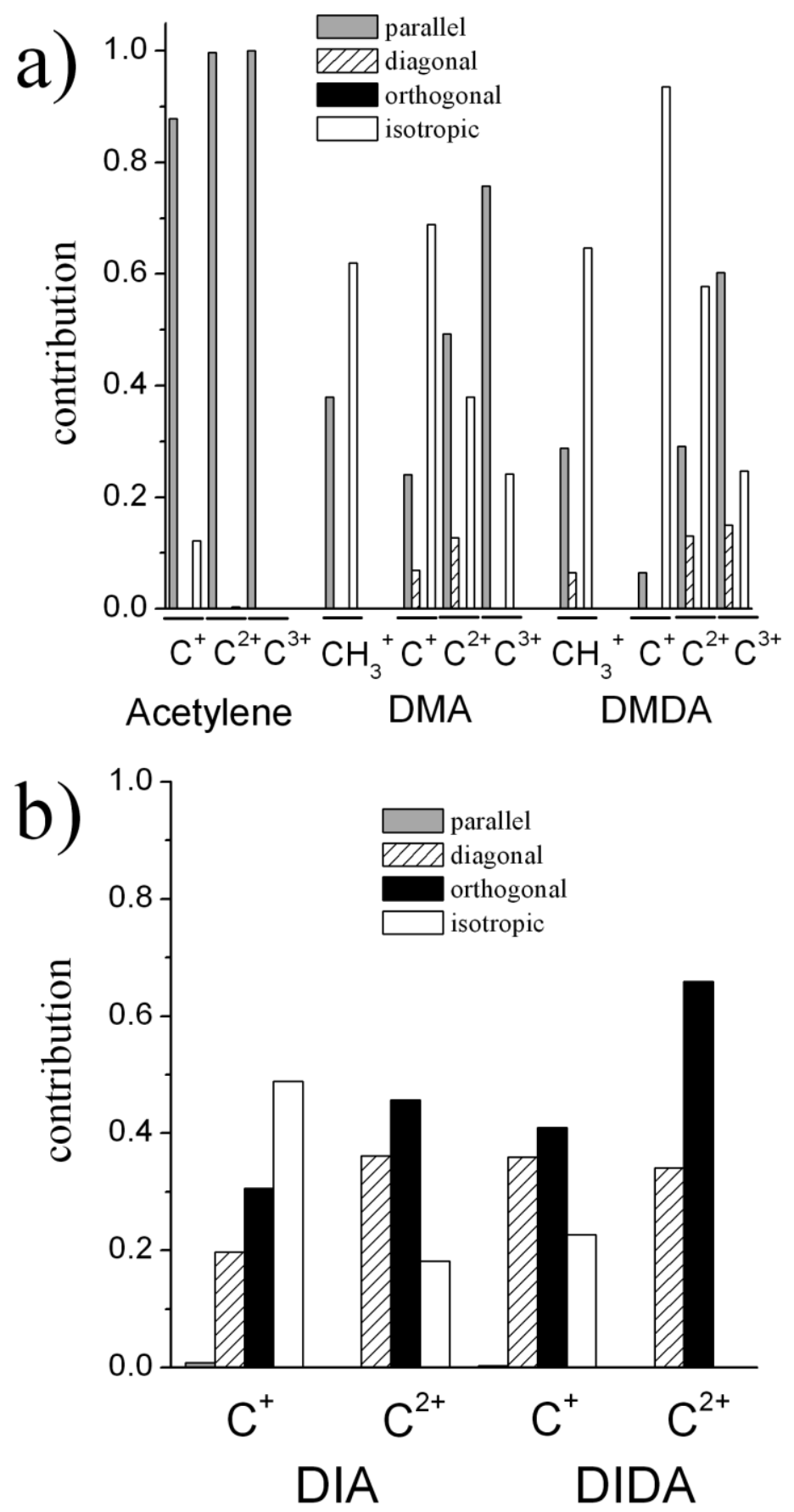

Fig. 7. The distribution of ion components ejected parallel, diagonal, orthogonal, and isotropic to the laser polarization direction from (a) acetylene, 2-butyne (DMA), and 2, 4-hexadiyne (DMDA), and (b) from diiodoacetylene (DIA) and diiododiacetylene (DIDA). The angular distributions of the carbon ions shown in Fig. 4 were fitted by Eq. (1). 


\section{References}

[1] Molecules and Clusters in Intense Laser Fields; Posthumus Ed.; Cambridge Univ. Press:

Cambridge, 2001.

[2] Yamanouchi, K. The Next Frontier. Science 2002, 295, 1659-1660.

[3] Mathur, D. Multiply Charged Molecules. Phys. Rep. 1993, 225, 193-272.

[4] Lammertsma, K.; Schleyer, P. von R.; Schwarz, H. Organic Dications: Gas Phase Experiments and Theory in Concert. Angew. Chem. Int. Ed. 1989, 28, 1321-1341.

[5] Ast, T.; Beynon, J. H.; Cooks, R. G. The Doubly-charged Ion Mass Spectra of Hydrocarbons. Org. Mass. Spectrom. 1972, 6, 749-763.

[6] Yatsuhashi, T.; Murakami, M.; Nakashima, N. Anisotropic Bulletlike Emission of Terminal Ethynyl Fragment Ions: Ionization of Ethynylbenzene-d under Intense Femtosecond Laser Fields. $J$. Chem. Phys. 2007, 126, 194316.

[7] Hishikawa, A.; Matsuda, A.; Fushitani, M.; Takahashi, E. J. Visualizing Recurrently Migrating Hydrogen in Acetylene Dication by Intense Ultrashort Laser Pulses. Phys. Rev. Lett. 2007, 99, 258302.

[8] Hishikawa, A.; Hasegawa, H.; Yamanouchi, K. Hydrogen Migration in Acetonitrile in Intense Laser Fields in Competition with Two-body Coulomb Explosion. J. Electron Spec. 2004, 141, 195-200.

[9] Xu, H.; Marceau, C.; Nakai, K.; Okino, T.; Chin, S. L.; Yamanouchi, K. Two Stages of Ultrafast Hydrogen Migration in Methanol Driven by Intense Laser Fields. J. Chem. Phys. 2010, 133, 071103.

[10] Xu, H.; Okino, T.; Nakai, K.; Yamanouchi, K.; Roither, S.; Xie, X.; Kartashov, D.; Zhang, L.; Baltuska, A.; Kitzler, M. Two-proton Migration in 1,3-Butadiene in Intense Laser Fields. Phys. Chem. Chem. Phys. 2010, 12, 12939-12942.

[11] Xu, H.; Okino, T.; Yamanouchi, K. Tracing Ultrafast Hydrogen Migration in Allene in Intense Laser Fields by Triple-ion Coincidence Momentum Imaging, J. Chem. Phys. 2009, 131, 151102. 
[12] Okino, T.; Watanabe, A.; Xu, H.; Yamanouchi, K. Ultrafast hydrogen scrambling in methylacetylene and methyl-d3-acetylene ions induced by intense laser fields. Phys.Chem.Chem.Phys. 2012, 14, 10640-10646.

[13] Nakashima, N.; Shimizu, S.; Yatsuhashi, T.; Sakabe, S.; Izawa, Y. Large Molecules in Highintensity Laser Fields. J. Photochem. Photobiol., C 2000, 1, 131-143.

[14] Frasinski, L. J.; Codling, K.; Hatherly, P. A. Covariance Mapping: a Correlation Method Applied to Multiphoton Multiple Ionization. Science 1989, 246, 1029-1031.

[15] Hishikawa, A.; Iwamae, A.; K. Yamanouchi, K. Ultrafast Deformation of the Geometrical Structure of $\mathrm{CO}_{2}$ Induced in Intense Laser Fields. Phys. Rev. Lett. 1999, 83, 1127-1130.

[16] Comstock, M.; Senekerimyan, V.; Dantus, M. Ultrafast Laser Induced Molecular Alignment and Deformation: Experimental Evidence from Neutral Molecules and from Fragment Ions. J. Phys. Chem. A 1993, 107, 8271-8281.

[17] Gibson, G. N.; Li, M.; Guo, C.; Nibarger, J. P. Direct Evidence of the Generality of Chargeasymmetric Dissociation of Molecular Iodine Ionized by Strong Laser Fields. Phys. Rev. A 1998, 58, 4723-4727.

[18] Hishikawa, A.; Iwamae, A.; Hoshina, K.; Kono, M.; Yamanouchi, K. Mass-resolved TwoDimensional Momentum Imaging of the Coulomb Explosion of $\mathrm{N}_{2}$ and $\mathrm{SO}_{2}$ in an Intense Laser Field. Chem. Phys. Lett. 1998, 3-4, 283-291.

[19] Sanderson, J. H.; Goodworth, T. R. J.; El-Zein, A.; Bryan, W. A.; Newell, W. R.; Langley, A. J.; Taday, P. F. Phys. Rev. A 2002, 65, 043403.

[20] Shimizu, S.; Zhakhovskii, V.; Sato, F.; Okihara, S.; Sakabe, S.; Nishihara, K.; Izawa, Y.; Yatsuhashi, T.; Nakashima, N. Shimizu, S.; Zhakhovskii, V.; Sato, F.; Okihara, S.; Sakabe, S.; 
Nishihara, K.; Izawa, Y.; Yatsuhashi, T.; Nakashima, N. Coulomb Explosion of Benzene Induced by an Intense Laser Field. J. Chem. Phys. 2002, 117, 3180-3189.

[21] Shimizu, S.; Zhakhovskii, V.; Murakami, M.; Tanaka, M.; Yatsuhashi, T.; Okihara, S.; Nishihara, K.; Sakabe, S.; Izawa, Y.; Nakashima, N. Coulomb Explosion of Hexa-fluorobenzene Induced by an Intense Laser Field. Chem. Phys. Lett. 2005, 4-6, 379-383.

[22] Kou, J.; Zhakhovskii, V.; Sakabe, S.; Nishihara, K.; Shimizu, S.; Kawato, S.; Hashida, M.; Shimizu, K.; Bulanov, S.; Izawa, Y.; Kato, Y.; Nakashima, N. Anisotropic Coulomb Explosion of $\mathrm{C}_{60}$ Irradiated with a High-intensity Femtosecond Laser Pulse. J. Chem. Phys. 2000, 112, 5012-5020.

[23] Cornaggia, C.; Schmidt, M.; Normand, D. Laser-induced Nuclear Motions in the Coulomb Explosion of $\mathrm{C}_{2} \mathrm{H}_{2}^{+}$Ions. Phys. Rev. A 1995, 51, 1431-1437.

[24] Yatsuhashi,T.; Mitsubayashi, N.; Itsukashi, M.; Kozaki, M.; Okada, K.; Nakashima, N. Persistence of Iodines and Deformation of Molecular Structure in Highly Charged Diiodoacetylene: Anisotropic Carbon Ion Emission. ChemPhysChem 2011, 12, 122-126.

[25] De, S.; Tezuka, H.; Bhatt, P.; Vesapidze, G.; Safvan, C. P.; Matsumoto, J.; Shiromaru, H. Do Linear Molecules Always Dissociate along Their Axis? Intra-molecular Scattering within Diiodoacetylene. J. Phys. Conf. Ser. 2015, 635, 032061.

[26] Nelson, D. J.; Blue, C. D.; Brown, H. C. Hydroboration Kinetics. 5. Kinetics of the Reaction of 9-Borabicyclo[3.3.1]nonane with Representative Haloalkynes in Carbon Tetrachloride. The Effect of Halogen Substitution upon the Stoichiometry and Rate of Hydroboration. J. Am. Chem. Soc. 1982, 104, 4913-4917.

[27] Luo, L.; Wilhelm, C.; Sun, A.; Grey, C. P.; Lauher, J. W.; Goroff, N. S. Poly(diiododiacetylene): Preparation, Isolation, and Full Characterization of a Very Simple Poly(diacetylene). J. Am. Chem. Soc. 2008, 130, 7702-7709. 
[28] Russo, M. V.; Sterzo, C. L.; Franceschini, P.; Biagini, G.; Furlani, A. Synthesis of Highly Ethynylated Mono and Dinuclear Pt(II) Tethers bearing the 4,4'-Bis(ethynyl)biphenyl (debp) Unit as Central Core. J. Organomet. Chem. 2001, 619, 49-61.

[29] Hankin, S. M.; Villeneuve, D. M.; Corkum, P. B.; Rayner, D. M. Intense-field Laser Ionization Rates in Atoms and Molecules. Phys. Rev. A 2001, 64, 013405.

[30] Tanaka, M.; Panja, S.; Murakami, M.; Yatsuhashi, T.; Nakashima, N. Intact Molecular Ion Formation of Cyclohexane and 2,3-Dimethyl-1,3-butadiene by Excitation with a Short, Intense Femtosecond Laser Pulse. Chem. Phys. Lett. 2006, 427, 255-258.

[31] Robson, L.; Ledingham, K. W. D.; McKenna, P.; McCanny, T.; Shimizu, S.; Yang, J. M.; ClaesGöran, W.; Lopez-Martensb, R.; Varju, K.; Johnsson, P.; Mauritsson, J. Volumetric Intensity Dependence on the Formation of Molecular and Atomic Ions within a High Intensity Laser Focus. J. Am. Soc. Mass Spectrom. 2005, 16, 82-89.

[32] Yatsuhashi, T.; Obayashi, T.; Tanaka, M.; Murakami, M.; Nakashima, N. Femtosecond Laser Ionization of Organic Amines with Very Low Ionization Potentials: Relatively Small Suppressed Ionization Features. J. Phys. Chem. A, 2006, 110, 7763-7771.

[33] Witzel, B.; Schro“der, H.; Kaesdorf, S.; Kompa, K.-L. Exact Determination of Spatially Resolved Ion Concentrations in Focused Laser Beams. Int. J. Mass Spectrom. Ion Processes 1998, 172, 229-238.

[34] Murakami, M.; Tanaka, M.; Yatsuhashi, T.; Nakashima, N. Enhancement of Anthracene Fragmentation by Circularly Polarized Intense Femtosecond Laser Pulse. J. Chem. Phys. 2007, 126, 104304.

[35] Feil S.; Głuch K.; Bacher, A.; Matt-Leubner, S.; Böhme, D. K.; Scheier, P.; Märk, T. D. Cross sections and ion kinetic energy analysis for the electron impact ionization of acetylene. $J$ Chem Phys. 2006, 124, 214307. 
[36] De, S.; Rajput, J.; Roy, A.; Ghosh, P. N.; Safvan, C. P. Ion-induced Dissociation Dynamics of Acetylene. Phys. Rev. A 2008, 77, 022708.

[37] Kloster-Jensen, E.; Pascual, C.; Vogt, J. Mass Spectrometric Studies of Mono- and Dihaloacetylenes. Helv. Chim. Acta. 1970, 53, 2109-2118.

[38] Pavičić, D.; Lee, K. F.; Rayner, D. M.; Corkum, P. B.; Villeneuve, D. M. Direct Measurement of the Angular Dependence of Ionization for $\mathrm{N}_{2}, \mathrm{O}_{2}$, and $\mathrm{CO}_{2}$ in Intense Laser Fields. Phys. Rev. Lett. 2007, $98,243001$.

[39] Ohmura, H.; Nakanaga, T. Quantum control of molecular orientation by two-color laser fields. J. Chem. Phys. 2004, 120, 5176-5180.

[40] Yatsuhashi T.; Nakashima, N.; Azuma, J. Coulomb Explosion of Dichloroethene Geometric Isomers at $1 \mathrm{PWcm}^{-2}$. J. Phys. Chem. A 2013, 117, 1393-1399.

[41] Moss, W. N.; Goroff, N. S. Theoretical Analysis of the ${ }^{13}$ C NMR of Iodoalkynes upon Complexation with Lewis Bases, J. Org. Chem. 2005, 70, 802-808.

[42] El-Sayed, M. A.; Tai, T. L. Kinetic Energy of Fragment Ions by Pulsed Laser-pulsed Extraction Field Technique and the Mechanism of Laser Multiphoton Ionization Dissociation: 2,4-Hexadiyne. $J$. Phys. Chem., 1988, 92, 5333-5337.

[43] Vékey, K.; Brenton, A. G.; Beynon, J. H. Comparison of $\left[\mathrm{C}_{6} \mathrm{H}_{6}\right]^{2+}$ Ion Structures: Application of Electron Capture Induced Decomposition to the Study of Isomers. Org. Mass Spectrom. 1989, 24, 3136.

[44] Herman, M.; Campargue, A.; El Idrissi, M. I.; Vander Auwera J. Vibrational Spectroscopic Database on Acetylene, $X^{1} \Sigma_{\mathrm{g}}{ }^{+}\left(\square{ }^{12} \mathrm{C}_{2} \mathrm{H}_{2},{ }^{12} \mathrm{C}_{2} \mathrm{D}_{2}\right.$, and $\left.{ }^{13} \mathrm{C}_{2} \mathrm{H}_{2} \square\right)$. J. Phys. Chem. Ref. Data, 2003, 32, 921-1361.

[45] Thomas, P. M.; Weber, A. High Resolution Raman Spectroscopy of Gases with Laser Sources. J. Raman Spectrosc. 1979, 8, 50-56. 
[46] Cornaggia, C. Carbon Geometry of $\mathrm{C}_{3} \mathrm{H}_{3}{ }^{+}$and $\mathrm{C}_{3} \mathrm{H}_{4}{ }^{+}$Molecular Ions Probed by Laser-induced Coulomb Explosion. Phys. Rev. A 1995, 52, R4328-R4331. 